\title{
Lamb wave-based air-coupled ultrasonic inspection technique for filament-wound composite pipes
}

\begin{abstract}
Filament-wound glass epoxy composite pipe-like structures require ultrasonic inspection in order to characterise any potential defects, such as those due to foreign inclusions and delaminations, so that defective as-manufactured components do not go into service. Current manual pointto-point contact or immersion-based ultrasonic inspections are effective but time consuming and expensive for largediameter/long-span composite pipes. This work addresses the need for rapid defect inspection for these composite pipes. The detection of multiple artificial inclusions in the pipe using the $A_{0}$ Lamb wave mode, generated and received using non-contact air-coupled ultrasonic transducers, is studied in this investigation. A two-step inspection technique is proposed. The first step consists of a single-sided global screening in the axial and radial directions of the pipe using the $A_{0}$ mode for rapidly locating the defects. In the second step, a limited-area point-to-point air-coupled throughtransmission inspection for sizing the defects is proposed. Lamb wave-based inspections are interpreted using a twodimensional intensity profile (B-scans), which satisfactorily indicates the approximate locations of defective regions. The aim of this study is also to demonstrate a system using this proposed Lamb wave technique.
\end{abstract}

Keywords: composite pipe, air-coupled ultrasonics, non-contact inspection, $\mathrm{A}_{0}$ Lamb mode.

\section{Introduction}

Due to the high specific strength, high specific modulus, corrosion resistance and high durability in areas such as energy, civil and defence applications, traditional metallic materials such as steel and aluminium are being replaced by polymer matrix composites.

Janardhan Padiyar $M$ received his Masters in Advanced Manufacturing Engineering in 2005 from the National Institute of Technology, Karnataka, India. He is pursuing his PhD at the Center for Nondestructive Evaluation (CNDE), Indian Institute of Technology, Madras (IIT-M). He is currently working at National Aerospace Laboratories Bangalore as a Scientist Fellow employed by the Council of Scientific and Industrial Research (CSIR), India. He has co-authored four papers in peer-reviewed journals and conference proceedings.

Krishnan Balasubramaniam* received his $P h D$ degree from Drexel University, Philadelphia, in 1989. Since 2000, he has been a Professor in the Department of Mechanical Engineering, Indian Institute of Technology, Madras, and also serves as Head of the Center for Nondestructive Evaluation. Before joining IIT-M in 2000, he was employed as an Associate Professor with the Department of Aerospace Engineering and Mechanics at Mississippi State University. He has over 327 technical publications (including 151 refereed journal papers).

The authors are with the Center for Nondestructive Evaluation and Department of Mechanical Engineering, Indian Institute of Technology Madras, Chennai, 600036, India.

*Corresponding author.Email: balas@iitm.ac.in
Filament winding is the most common manufacturing process for producing cylindrical composite structures. The filament winding process produces parts by first winding a fibre tow, which is passed through a resin bath to impregnate the tow of fibres, and then wrapping it around a removable mandrel and curing it to form the composite structure. Components typically made by this process include pipes for the transfer of fluids, oxygen storage vessels, hydrogen fuel tanks, missile canisters and pressure vessels ${ }^{[1,2]}$. During the manufacture of these components, dry patches due to insufficient epoxy resin or foreign objects, such as paper, may accidentally be embedded. This results in the formation of defects, such as delaminations. The foreign object inclusions and other defects, such as delaminations, are sub-surface in nature and hence cannot be detected through visual inspection. Ultrasonic nondestructive testing (NDT) methods have been used to locate such defects. Shorter-length diameter pipes can be inspected mostly by water immersion $\mathrm{C}$-scan systems, while larger lengths use squirter (water-jet)-based expensive C-scan systems. Conventional $\mathrm{C}$-scan inspection using a bulk wave is a point-by-point inspection and is, therefore, a time consuming and uneconomical method for application to low-cost glass epoxy composite pipes. The manufacture of high-value filament wound products, such as canisters and pressure vessels, is carried out in several stages, with in-process inspection techniques needed at each stage. Hence, there is a need to devise newer methodologies for economical and rapid inspection in a production environment for filament-wound composite pipe-like structures.

Several guided wave-based non-destructive inspection techniques using different types of guided wave modes have been developed for the screening of defects in the axial and circumferential directions of metallic pipes. The major benefits of guided waves are their ability to inspect the entire cross-sectional area of the structure and that they can propagate long distances, giving these waves the capability of rapid global screening of full-scale pipe structures. Most of these guided wave-based inspections of metallic pipes have been carried out using contact-based transducers, mounted on wedges, operating in pulse-echo mode ${ }^{[3-4]}$. Inspection with a guided wave ultrasonics pulse-echo transducer configuration in composite pipes is quite difficult due to the inherent material attenuation and the different types of defect. Since the composite pipes are made of orthotropic material, specific mode-based Lamb wave inspection using contact transducers requires designing of different wedges. A potential way out of these problems is to use couplant-free/noncontact transducers during inspection. Due to the attenuation in composites, the ability to extract reflected signals from defects is diminished and a pitch-catch approach is needed for the application of guided waves in composite pipes.

In recent years, air-coupled ultrasonic transducers have become a commercial success in industry for the transmission and reception of bulk waves and also Lamb waves in thin composite structures. The principle of air-coupled ultrasonic generation, propagation and detection of Lamb waves in pitch-catch mode has been extensively discussed in the literature ${ }^{[5-7]}$. Air-coupled transducers could be used easily with an automated scanning system, when compared to 
contact-based ultrasonic transducers. They also have the ability of couplant-free transduction of Lamb modes along with repeatability of measurements, when compared to the wedge-based Lamb wave generation technique. Improvements in air-coupled ultrasonic transducer materials using 1-3 piezocomposite low-impedance gas matrix probes (GMP) have made it possible to acquire signals at a higher signal-to-noise ratio than earlier bulk piezoelectric transducers ${ }^{[8]}$, thus reducing the need for larger number averaging of the signal during inspection. The ability of GMP-based aircoupled transducers to penetrate higher thickness composites and metals has opened new areas of applications for air-coupled NDT ${ }^{[9]}$. However, applications of air-coupled ultrasonics have been limited in the frequency range of 50 to $500 \mathrm{kHz}$ due to the high attenuation of ultrasonic waves within air and in composites. The single-sided air-coupled ultrasonic inspection technique using the $\mathrm{A}_{0}$ Lamb wave mode has received considerable interest from researchers and new applications have been proposed for the detection and visualisation of defects in plate-like composite structures ${ }^{[10,11]}$. The single-sided Lamb wave-based techniques have also been used successfully for inspecting aerospace-grade honeycomb structures and impact damage in composite wind turbine blades ${ }^{[12,13]}$. Filament-wound composite-wrapped pressure vessels have been inspected for damage using guided waves generated by air-coupled transducers, and the defects were located using the amplitude changes of the guided wave mode ${ }^{[14]}$.

A novel pitch-catch scanning approach using two immersion transducers for transmission and reception of the $S_{0}$ Lamb wave mode was proposed to screen impact-induced delaminations in composite plate ${ }^{[15]}$. This inspection approach required scanning in two orthogonal directions to detect and locate the damage. The changes in the wave velocity and amplitude of the signal due to delaminations were used as the damage detection criteria. Other researchers have shown interest in the application of $A_{0}$ Lamb wave mode-based B-scan imaging, using air-coupled transducers for finding interface delaminations in a composite T-joint ${ }^{[16]}$. It was shown that it is possible to identify the defects and estimate the approximate delamination dimensions from ultrasonic B-scans. In the present study, it is interesting to consider the application of Lamb waves for the inspection of composite pipes (curved structures) with subsurface defects due to thin inclusions between the adjacent layers. However, to the authors' knowledge, there are no studies for screening of inclusions in a composite pipe using guided waves generated by air-coupled transducers. The main objective of this paper is to investigate the feasibility of developing a non-contact inspection system for the inspection application of filament-wound pipe, by combining Lamb wave-based B-scan imaging for global screening with point-to-point $\mathrm{C}$-scan imaging for sizing of foreign inclusions. A secondary objective is to demonstrate the potential of this preliminary work to reduce the inspection time using the proposed scanning approach. This would lead towards developing a practical industrial application.

This paper is organised as follows: Section 2 discusses the specimen and experimental set-up developed for the current study; Section 3 outlines the rapid inspection technique adopted for defect detection in the composite pipe and presents the experimental results; and conclusions are summarised in Section 4.

\section{Experimental technique}

\subsection{Specimen details}

A single glass fibre-reinforced plastic (GFRP) pipe specimen was fabricated using 10 layers of glass fibre with an epoxy matrix. The lay-up consisted of 10 windings at $55^{\circ}$ to produce a thickness of $6 \mathrm{~mm}$ and a fibre volume ratio of $68 \%$. The pipe dimensions were $500 \mathrm{~mm}$ length and $300 \mathrm{~mm}$ internal diameter. Four different sizes of grease-coated Teflon film, $0.2 \mathrm{~mm}$ thick with sizes of $15 \times 15 \mathrm{~mm}^{2}, 20 \times 20 \mathrm{~mm}^{2}, 25 \times 25 \mathrm{~mm}^{2}$ and $30 \times 30 \mathrm{~mm}^{2}$ (named
A, B, C and D), were inserted to simulate artificial delaminations representing foreign object inclusions, which may be introduced during manufacturing. These films were placed at various locations along the length in the fourth layer from the outer surface of the pipe. However, there was no bulging of the pipe due to these inclusions. The locations and sizes of these defects are shown in Figure 1. However, it should be noted that the Teflon film insert may move a few millimeters during the winding process and the actual defect orientation may be slightly different from the expected placement.

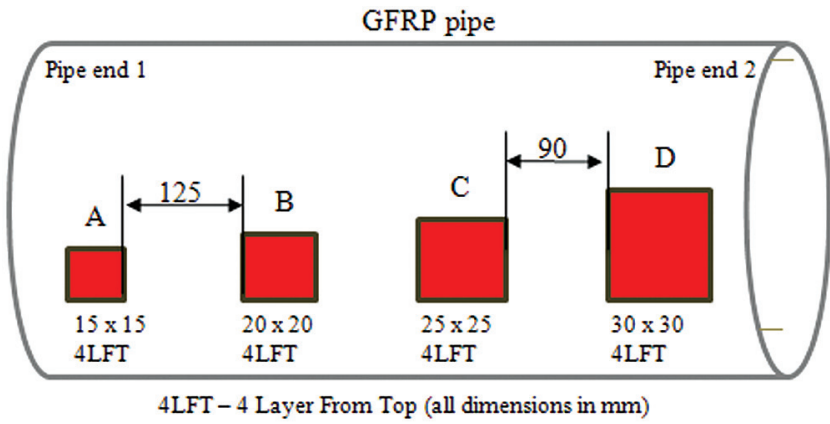

Figure 1. Schematic representation of the GFRP pipe with foreign inclusions

\subsection{Air-coupled Lamb wave generation and group velocity measurement}

\subsubsection{Air-coupled transducer}

Air-coupled ultrasonic transducers from Ultran group, using gas matrix piezo (GMP) materials, were selected for the experiments reported in this paper. These types of transducer have a low acoustic impedance and higher efficiency than the older-generation bulk piezo-based air-coupled transducers. Due to the strong attenuation of ultrasonic waves at higher frequencies, both in air and in the composite material, lower-frequency transducers were chosen for the current experiments. The transducers selected were $40 \mathrm{~mm}$-square and cylindrically-focused with a narrow bandwidth, working at a central frequency of $100 \mathrm{kHz}$. Air-coupled ultrasonic inspection does not need an immersion tank or a squirter-based C-scan system, hence it is more economical with simpler stepper motor drives. All Lamb wave experiments in the present study were conducted in pitch-catch mode.

\subsubsection{Selective generation of a Lamb wave mode}

The diameter-to-thickness ratio of the present pipe specimen was 52 , hence low-frequency ultrasonic wave propagation in this pipe can be approximated to a Lamb wave propagation in a composite plate of the same thickness. Lamb waves propagating in a plate are made up of a combination of compression (longitudinal) and shear displacements. These waves are classified into symmetric and anti-symmetric Lamb modes, depending on the through thickness displacement profile. The symmetric and anti-symmetric family of Lamb modes are denoted by $S_{0}, S_{1}$, etc and $A_{0}, A_{1}$, etc. The type of Lamb wave modes generated in a plate depends on both the excitation frequency and plate thickness, as well as the angle of incidence of the transducers. However, at a lower value frequencythickness product, only the fundamental Lamb wave modes, ie $\mathrm{S}_{0}$ and $A_{0}$, can propagate in the plate. The symmetric Lamb wave $S_{0}$ mode has predominantly in-plane particle motion and the $\mathrm{A}_{0}$ mode has predominantly out-of-plane particle motion. The $\mathrm{A}_{0}$ Lamb mode was chosen as the excitation mode in this work because it has the highest coupling efficiency when used along with air-coupled ultrasonic transduction. The transducer orientation angle for efficient transmission and reception of the $\mathrm{A}_{0}$ mode into a structure can be determined from the $\mathrm{A}_{0}$ mode phase velocity in the material at a particular frequency and the velocity of sound in air (SnellDescartes Law).

Generally, lay-up and material properties are varied by the 
manufacturer of filament-wound composite pipes and are not the same for all pipes. It is not practical to know the exact properties of each pipe material under inspection, so it is necessary to determine, by trial, the optimum transducer angle for efficient excitation and reception of the $\mathrm{A}_{0}$ mode. To accomplish this, the transducer was first positioned in a pitch-catch configuration over the pipe, to generate a Lamb wave propagating axially in the pipe. In pitchcatch configuration, two transducers are on the same side of the pipe separated by a known distance. The transducers were inserted into a custom-made plastic fixture to reduce the weight attached to the scanner (details are given in the next section). A $-900 \mathrm{~V}$ pulse driving the transmitter and the receiver with a total gain of $60 \mathrm{~dB}$ was used. To find the optimum transducer angle, both the transmitter and receiver angle were varied in tandem until the maximum signal amplitude was obtained; in this case, at an angle of $20^{\circ}$. The unaveraged $\mathrm{A}_{0}$ mode propagating for a distance of 88 $\mathrm{mm}$ in the $6 \mathrm{~mm}$-thick pipe is shown in Figure 2(a). Due to the lower signal-to-noise ratio, averaging and filtering of 64 received signals was performed and a sample A-scan signal is shown in Figure 2(b). The Fourier transform of this averaged signal is shown in Figure 2(c) and gives the transducer frequency response, which shows a narrow bandwidth centred at $100 \mathrm{kHz}$.

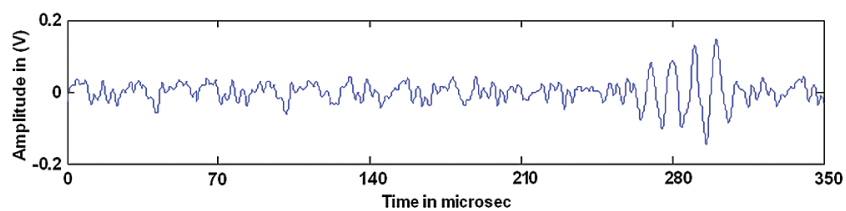

(a)

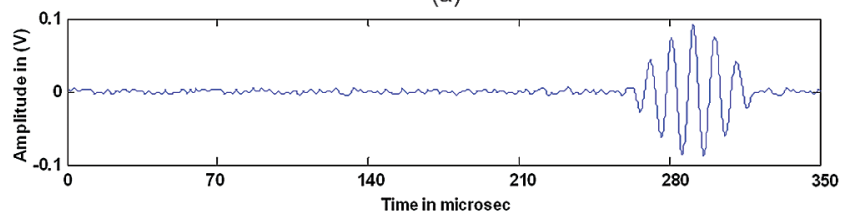

(b)

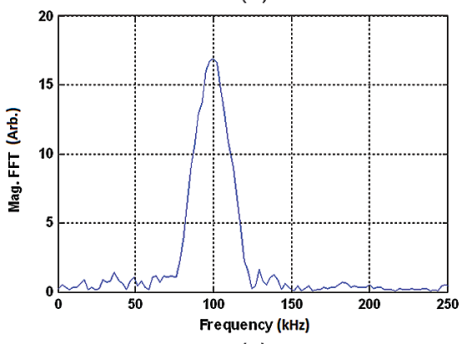

(c)

Figure 2. (a) Unaveraged time domain signal (A-scan) received by cylindrically-focused air-coupled receiver; (b) averaged (64 times) A-scan; (c) the frequency spectrum of averaged A-scan (the transducer separation distance maintained at $88 \mathrm{~mm}$ )

\subsubsection{Group velocity measurement}

To find the effect of the angle of the fibre wound on the pipe on the Lamb wave velocity, a group velocity measurement was carried out. The transmitter and receiver were oriented at the $\mathrm{A}_{0}$ mode angle, as described in the previous section, the receiver was moved to position R1, as indicated in Figure 3, and an A-scan was recorded. The receiver was then moved to position $\mathrm{R} 2$ and a similar measurement was carried out. The arrival times of the $\mathrm{A}_{0}$ mode at $\mathrm{R} 1$ and $\mathrm{R} 2$ are denoted as $\mathrm{t} 1$ and $\mathrm{t} 2$, respectively (these times are determined by taking the peak of the envelope fitted over each signal received at position $\mathrm{R} 1$ and $\mathrm{R} 2$ and measuring the time difference). The following expression gives the group velocity: $\mathrm{Cg}=(\mathrm{R} 2-\mathrm{R} 1) /(\mathrm{t} 2-\mathrm{t} 1)$. A similar procedure was repeated for the transducers placed around the circumference to obtain the group velocity in the radial direction, and the results are shown in Table 1. From Table 1, the effect of the angle of the fibre wound on the pipe on the Lamb wave velocity is clearly seen.

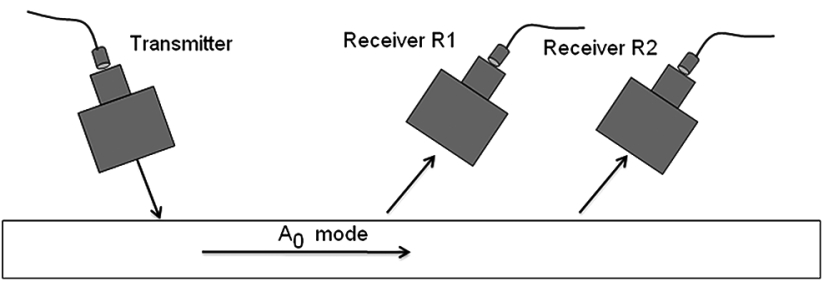

Figure 3. Transducer configuration for the $A_{0}$ mode group velocity measurement

Table 1. Experimental measurement of $A_{0}$ mode group velocities

\begin{tabular}{|c|c|}
\hline $\begin{array}{c}\text { Axial direction group velocity } \\
\mathrm{m} / \mathrm{s}\end{array}$ & $\begin{array}{c}\text { Radial direction group velocity } \\
\mathrm{m} / \mathrm{s}\end{array}$ \\
\hline 1818.2 & 1972.4 \\
\hline
\end{tabular}

\subsection{Evaluation of feasible transducer separation distance}

The ultrasonic attenuation of Lamb waves in glass fibre composites is very large when compared to other composites, such as carbon fibre-reinforced plastic (CFRP). Since the proposed work is for industrial application, where GFRP pipes of different diameters and length are manufactured, it is required to find the feasible transducer separation distance along the axial and radial directions. In order to obtain a higher signal-to-noise ratio (SNR) by a factor of 3, averaging of the individual signal is needed, which in turn results in longer inspection times. It was observed that software averaging of 64 A-scan signals and recording of a single full waveform takes about $8 \mathrm{~s}$. The following procedure was used to find the feasible transducer separation distance for propagation along the axial direction. The transmitter and receiver were oriented to generate and receive the $\mathrm{A}_{0}$ mode along the axial direction, with an initial separation distance of $100 \mathrm{~mm}$. By exciting the transmitter with a $-900 \mathrm{~V}$ input signal and using a $60 \mathrm{~dB}$ receiver gain, the amplitude of the $\mathrm{A}_{0}$ mode was measured. The receiver was moved incrementally in the axial direction. For each $10 \mathrm{~mm}$ movement of the receiver, one A-scan was captured. Figure 4 shows the trend in the received amplitude of A-scan signals as the separation distance between the transducers increased. Similar measurements were carried out along the radial direction. The variation of amplitude with transducer separation distance is plotted in Figure 4. It was also observed that the received signal dropped down to less than $0.1 \mathrm{~V}$ when the transducer separation distance was increased to $325 \mathrm{~mm}$ or more in the radial direction. Using the amplitude information from these measurements, the pipe area to be scanned was partitioned for circumferential and axial scanning into three radial segments and two axial regions, respectively, as shown in Figure 5. Depending on the size, length and material attenuation of a composite pipe, similar Lamb wave measurements can be carried out for other pipe sizes.

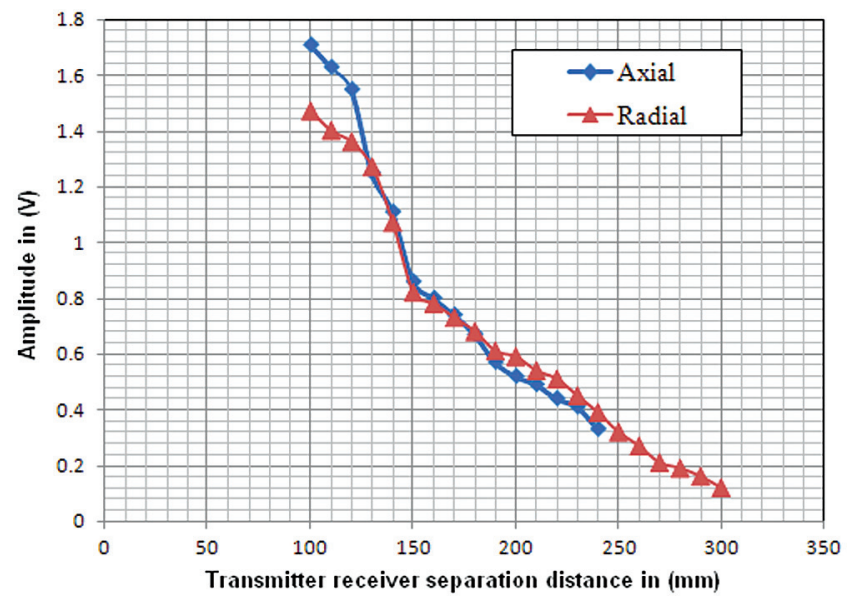

Figure 4. Variation of the received A-scan amplitudes in the axial and the radial directions with increase in transducer separation 


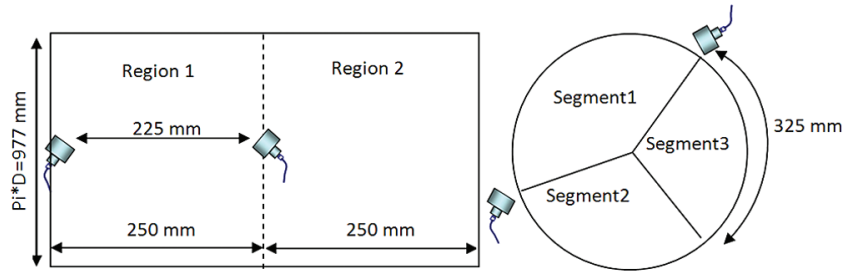

Figure 5. Pipe surface partitioned during scanning into two axial regions and three radial segments along the circumference of the pipe

\subsection{Experimental instrumentation}

Figure 6 shows the schematic of the experimental set-up used for Lamb wave velocity measurements and Lamb wave scanning of the pipe using a stepper motor-controlled scanner. This set-up allows the waves to travel either in the axial or radial direction. This scanner was used to rotate the pipe around its axis and translate the transducer attachment axially (not shown in the schematic). The transmitter was excited by an electrical spike of $-900 \mathrm{~V}$ using a Panametrics 5058 pulser/receiver; this generates an ultrasonic pressure wave in the surrounding air. The pulser/receiver damping was set as per the transducer manufacturer recommendation and the attenuator was set to zero. The pressure wave generated by the transmitter propagates through the air and generates an $\mathrm{A}_{0}$ Lamb mode in the pipe. The $\mathrm{A}_{0}$ mode propagates through the thickness of the pipe parallel to the pipe axis. Some of this energy leaks into the surrounding air where it is picked up by the receiver to generate an electrical signal. The received signal was amplified in stages, initially by a low-noise preamplifier in the pulser/receiver $(20 \mathrm{~dB})$ and subsequently by a $40 \mathrm{~dB}$ preamplifier. Furthermore, the pulser/ receiver was set to filter the signal using a band-pass filter with a lower cut-off frequency of $50 \mathrm{kHz}$ and an upper cut-off frequency of $500 \mathrm{kHz}$. This filtered signal was then acquired by a high-speed 12-bit analogue-to-digital converter (ADC) having a sampling frequency of $10 \mathrm{MHz}$ (a National Instruments PXI card on a PXI 1033 Chassis), and subsequently transferred to a PC for storage. A custom LabVIEW ${ }^{\mathrm{TM}}$ (Laboratory Virtual Instrument Engineering Workbench)-based data acquisition and signal processing program was used to perform filtering and averaging of the acquired data to improve the SNR of the digitised data.

\section{Lamb wave-based rapid inspection procedure}

The procedure proposed in the present study for the rapid detection, location and sizing of inclusion areas in pipes consists of a twostep procedure: a Lamb wave-based rapid inspection followed by

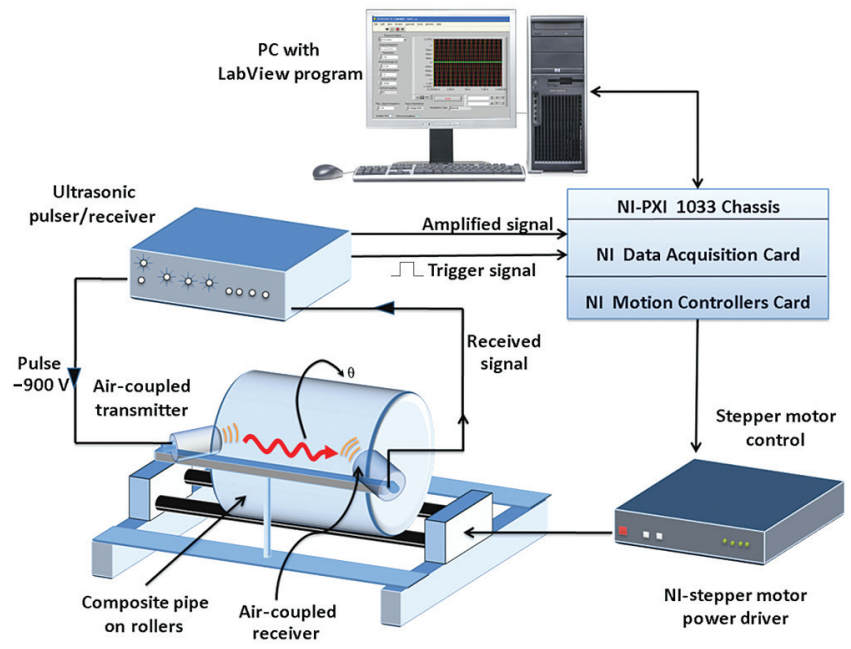

Figure 6. Schematic illustration of instrumentation set-up of the air-coupled inspection system conventional point-to-point through-transmission scanning. To locate the defects within the pipe wall, the inspection was carried out by propagating the waves axially, during which the pipe incrementally revolves around its axis, followed by propagating the waves radially and incrementally moving the transducer attachment parallel to the pipe axis. After locating the defects in both directions, sizing was carried out only at the suspected defect locations using a bulk wave air-coupled through-transmission C-scan.

\subsection{Experimental results: $B$-scans}

\subsubsection{Axial measurements}

Lamb wave axial measurements were performed using the ultrasonic instrumentation system described earlier with the transducers mounted in a pitch-catch configuration, as shown in Figure 7. The transducers were oriented at $20^{\circ}$ to the surface normal with a separation distance fixed at $225 \mathrm{~mm}$ to accommodate the finite size of the transducer (the length of region 1 was $250 \mathrm{~mm}$ ). Scanning was carried out as described above using a step size of $1^{\circ}$ along the pipe axis. The record length of the captured A-scan signals was fixed at $300 \mu \mathrm{s}$ (3000 points) at $10 \mathrm{MHz}$ sampling frequency, so that the record length was more than the time taken by the $\mathrm{A}_{0}$ mode to travel from the transmitter to the receiver, including the air paths. The $\mathrm{A}_{0}$ mode propagation between the transducers was partially blocked, depending on the defect orientation in the pipe. Due to this effect, there was an amplitude variation in the A-scan signals. Similar trials were repeated for region 2. A-scans obtained over regions 1 and 2 were further processed into B-scans. The B-scan representation in this work consists of individual Hilbert transforms of A-scans of the Lamb wave arranged adjacent to each other (the term B-scan used here should not be confused with ultrasonic bulk wave terminology, which represents cross-section imaging). The B-scan in Figure 8 shows the scan length in millimetres as the vertical axis and the horizontal axis showing the enveloped Hilbert transform time domain signals. The B-scan colour coding represents the signal amplitude. Thus, axially propagating waves give the defect locations around the pipe circumference. It can be seen from the B-scan results in Figures 8(a) and 8(b) that the defect is between $600 \mathrm{~mm}$ to $700 \mathrm{~mm}$; this corresponds to the end of the second segment. Figure 9 shows A-scans captured at three different regions with respect to the transmitter-receiver centreline: (a) a good region (blue); (b) when the defect is in line with the transducers (the inclusion area); and (c) when the defect edge is in line with the transducers (shadow region). The signal amplitude in the good region was $50 \%$ higher than in the other two regions. When the defect was in line with the transducers, the $\mathrm{A}_{0}$ mode undergoes reflections and mode conversion; this results in a signal of very low amplitude. In addition to this issue, there was an increase in arrival time due to a local change in thickness near the defect. The axial B-scans give the defect locations around the circumference, which further indicates that radial scanning is

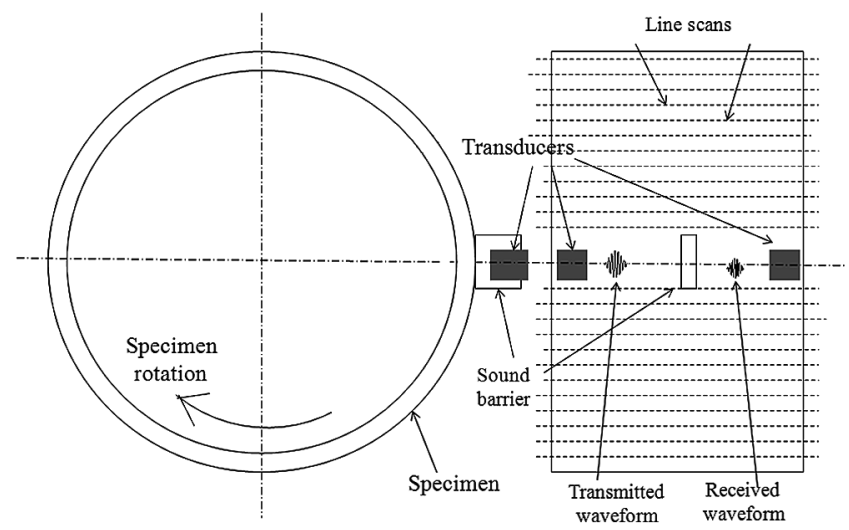

Figure 7. Scanning direction during axial measurements; transducer attachment is stationary with rotation of the pipe 


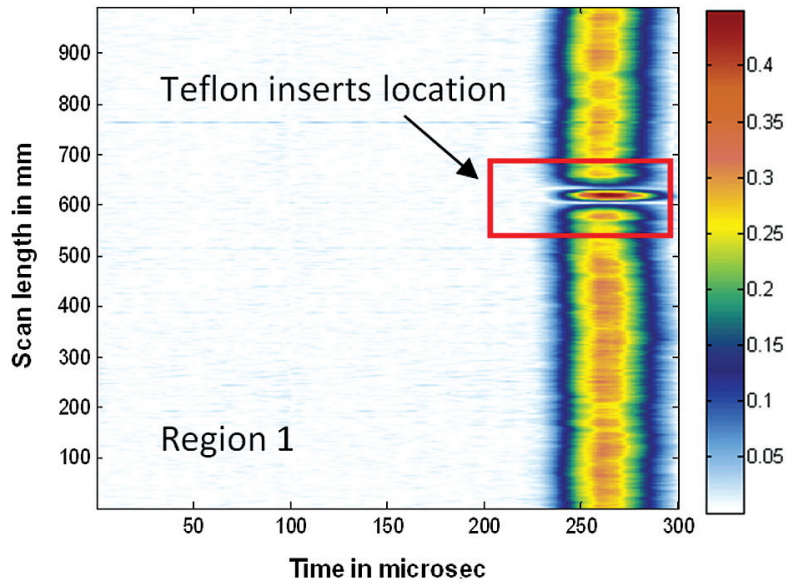

(a)

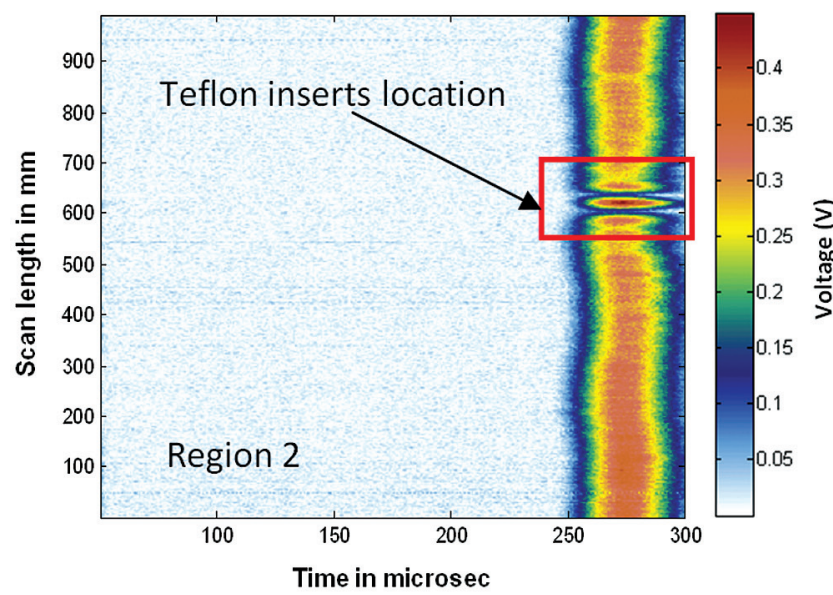

(b)

Figure 8. B-scan image results obtained by separating the axial length of the pipe into two regions, each $250 \mathrm{~mm}$ in length: (a) region 1; and (b) region 2

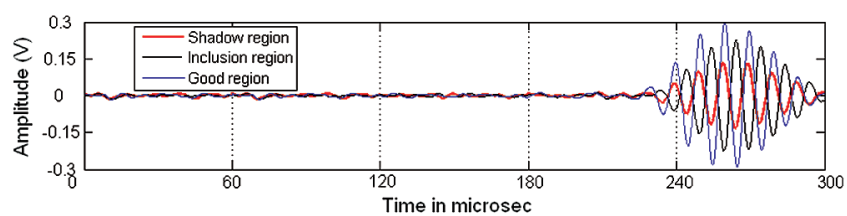

Figure 9. Comparison of A-scans at three different points when the transducers are at different positions along the Teflon inclusion identified in the B-scan in Figure 8(a)

necessary only in the segment of the composite pipe that falls in the location of the second segment, as shown in Figure 5. The total scanning time taken to carry out line scans in both regions 1 and 2 , with an averaging of 64 samples, was around $4.4 \mathrm{~h}$ (for a single region, each acquisition is 977 horizontal lines with one waveform being captured for each line).

A separate experiment has been conducted to show the possibility of long-range inspection with air-coupled transducers covering both regions 1 and 2, as shown in Figure 10(a). However, it was observed that, due to an increase in the distance between the transducers, the SNR is lowered and higher averaging of 256 times was needed to acquire measurable signals, which in turn increases the time needed for scanning and hence is counterproductive to the aim of the proposed technique. The B-scan results shown in Figure 10(b) from this experiment indicate the presence of a discontinuity.

\subsubsection{Radial measurements}

Since measurements using axially propagating waves have already given the approximate location of the inclusion areas around the circumference in segment 2, radial scanning was also carried out in segment 2. However, radial measurements are not necessary if

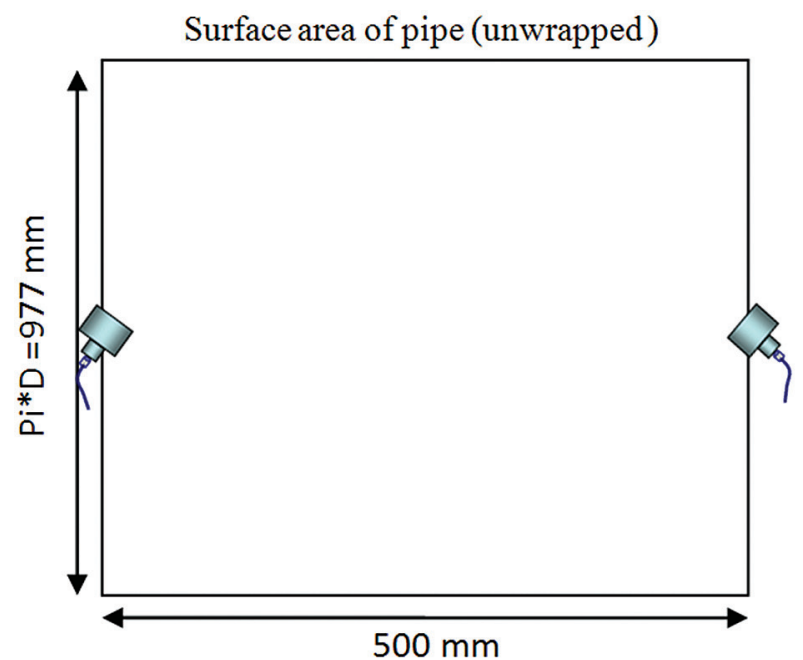

(a)

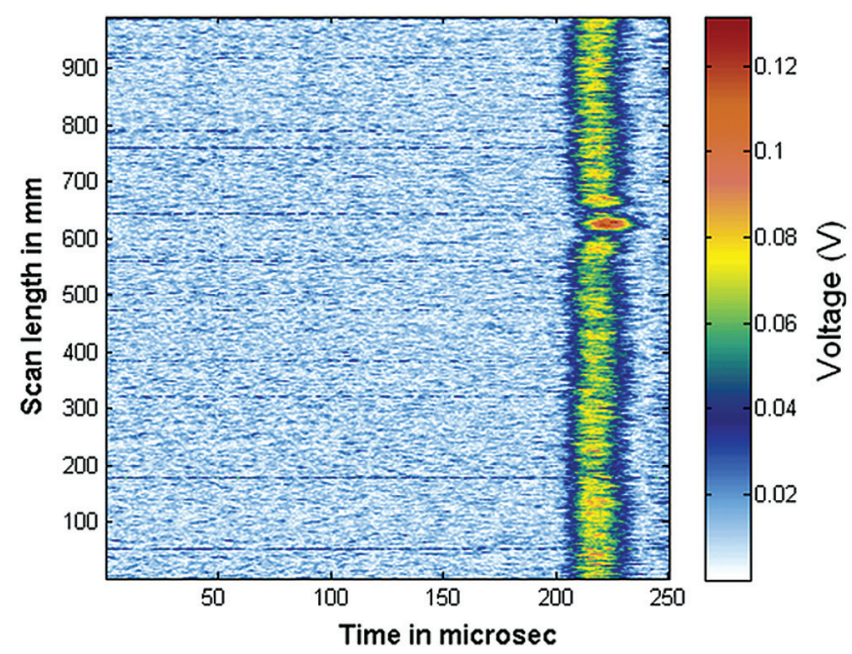

(b)

Figure 10. (a) The unwrapped pipe surface area; (b) B-scan image results obtained by covering the whole axial length of the pipe $(450 \mathrm{~mm})$, showing the feasibility of long-range inspection in composites

there no is indication of an inclusion in the axial measurements. Figure 11 shows the schematic of the locations of the air-coupled transducers for taking measurements using radially-propagating waves. Again, transducers were operated in the pitch-catch mode to generate and receive the $\mathrm{A}_{0}$ Lamb wave. The transducer separation distance and air column height (between the transducer and pipe) were $325 \mathrm{~mm}$ and $20 \mathrm{~mm}$, respectively. Now, the record length of captured A-scan signals was fixed at $350 \mu$ s (3500 points). The aircoupled transducer attachment shown in Figure 12(a) was moved axially, with data being captured every $1 \mathrm{~mm}$. A similar procedure

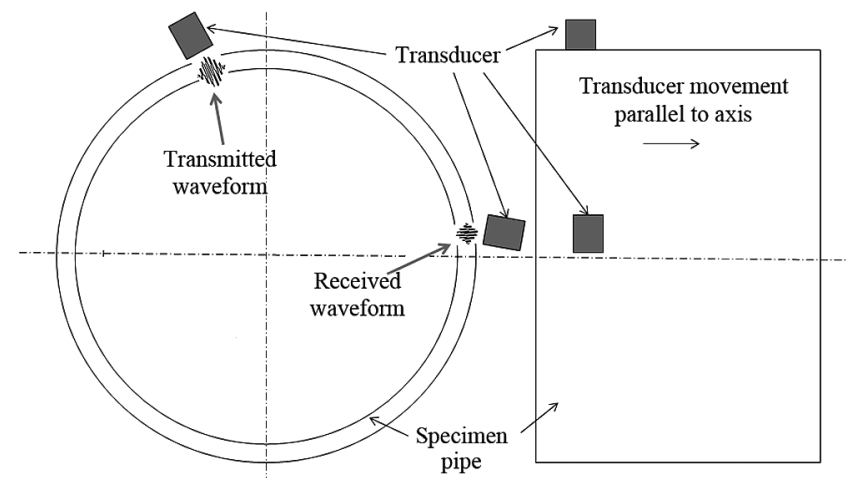

Figure 11. Scanning direction during radial measurements; transducer arrangement moves axially and the pipe is stationary 


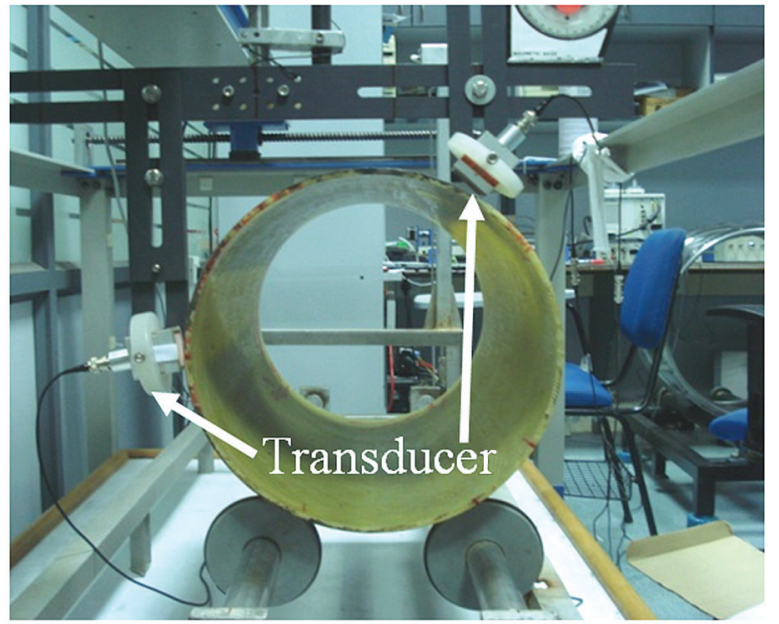

(a)

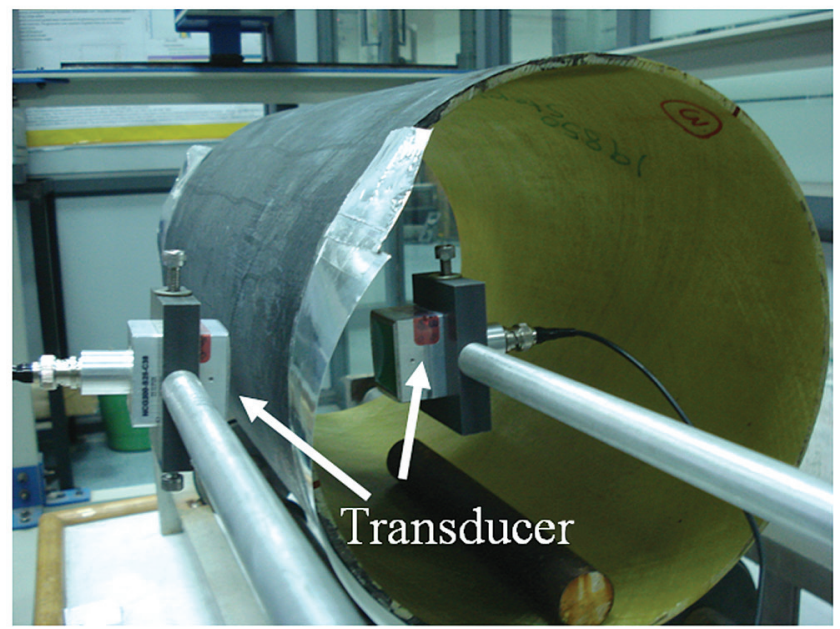

(b)

Figure 12. Pictorial view of the air-coupled transducer configuration used during (a) radial configuration and (b) normal incidence through-transmission

was repeated for segments 1 and 3. The results of these radial scans for all three segments are shown as B-scans in Figures 13(a), 13(b) and 13(c). Among the radial B-scans shown in Figure 13, Figure 13(b) gives the defect locations along the length of the pipe. Thus, the time taken for line scanning of a single radial segment using 64 averages is about one hour. During manufacture of the pipe, there was apparently an unintended defect introduced at the pipe edge; this defect showed up in segments 1 and 3, as seen in Figure 13. This defect was not investigated further. Thus, the proposed Lamb wave technique for rapid defect location will take a total time of about $5.4 \mathrm{~h}$. However, the software averaging and storing of the full waveform signals increases the time needed during the Lamb wave scan, hence we believe that if the averaging of the signals is carried out through a dedicated hardware, then the time of the inspection may reduce considerably.

\subsection{Experimental results: air-coupled through- transmission C-scan measurements}

To quantify the size of defects identified in segment 2 , an air-coupled through-transmission point-to-point ultrasonic C-scan was carried out using transducers having a frequency of $200 \mathrm{kHz}$ at a spatial resolution of $1 \mathrm{~mm}$. These transducers were mounted coaxially, such that the transmitter was on the outer side and the receiver was on the inner side of the pipe, as shown in Figure 12(b). During this experiment, the ultrasonic pulses were transmitted through the pipe wall and detected by the receiver. The peak signal amplitude of the transmitted ultrasonic pulse was used to form the C-scan

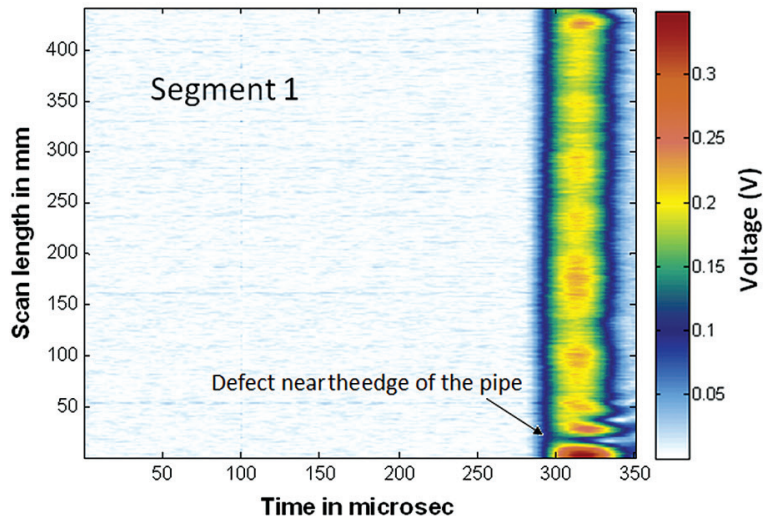

(a)

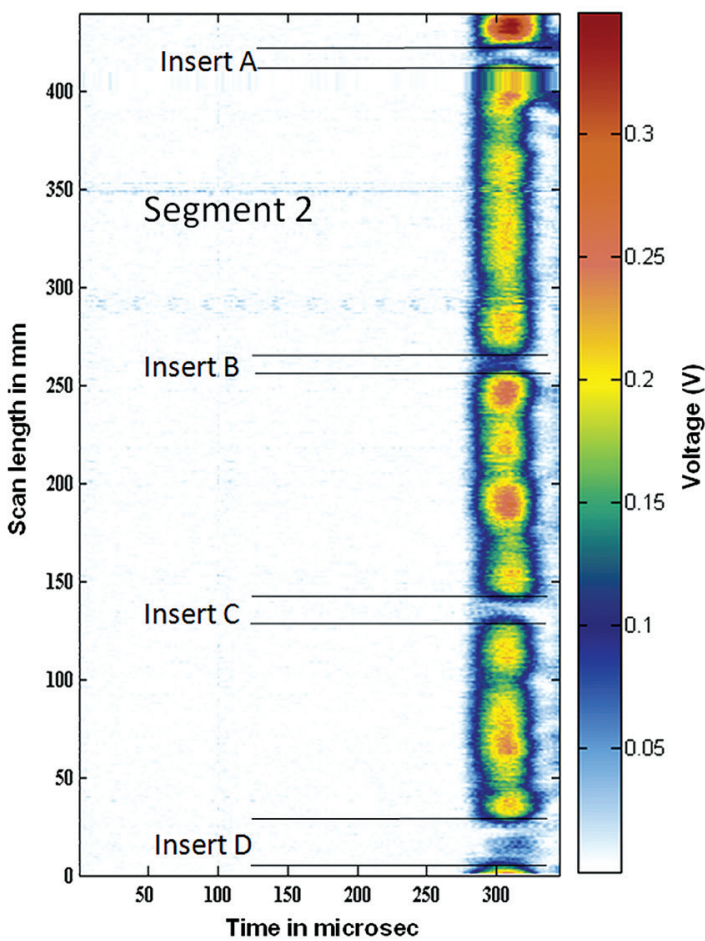

(b)

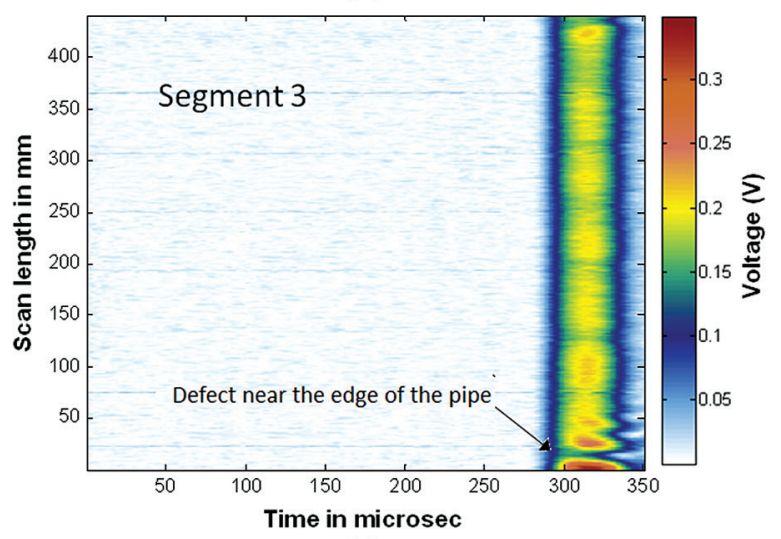

(c)

Figure 13. B-scan imaging results obtained for the three radial segments of the pipe: (a) segment 1; (b) segment 2; and (c) segment 3

image. The axial scanning length was limited by the lab scanning apparatus and hence the through-transmission C-scan was carried out first in region 1 and then in region 2. C-scan images of the four inclusion areas are shown in Figures 14(a) and 14(b). As seen in these Figures, the ultrasonic signal is of very low amplitude at the defect area; the received signal amplitude was mapped (lowest to highest) from red to black. The dark red squares in the $\mathrm{C}$-scan reveal 
the positions of the simulated defects within the pipe. However, the edge of the defect was found to be distorted. This was probably due to diffraction effects at the defect edges. The measured defect areas are in good agreement with the actual values. The time taken with our laboratory set-up for a through-transmission C-scan of region 1 of the pipe, with $1 \mathrm{~mm}$ resolution and a scanning speed of $500 \mathrm{~mm} / \mathrm{min}$, was about $8 \mathrm{~h}$ (at $1 \mathrm{~mm}$ resolution, each C-scan requires 977 scan lines, with 250 values of the amplitude being captured for each line). This shows that a conventional C-scanbased approach would take much more time for the entire pipe specimen than the guided wave approach. Hence, a C-scan is recommended to be used only for the purpose of defect sizing, once the defect locations are determined using the relatively quick Lamb wave-based line scan technique proposed in this paper.

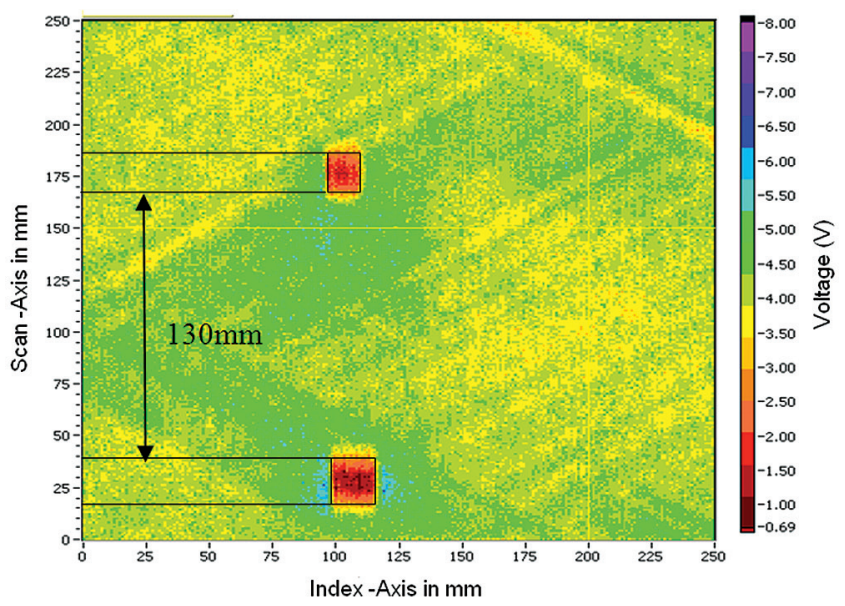

(a)

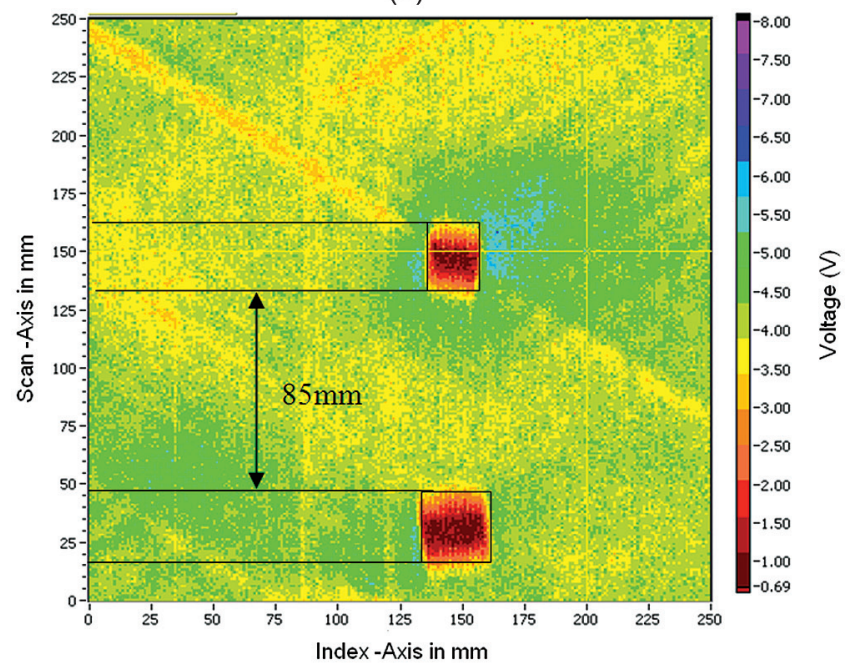

(b)

Figure 14. Normal incidence through-transmission C-scans in the second segment of the pipe using a $100 \mathrm{kHz}$ air-coupled ultrasonic transducer: (a) region 1; and (b) region 2

Since the artificial inclusions were rectangular in size, the inclusion width from the Lamb wave B-scan of segment 2, shown in Figure 13(b), could be estimated by measuring the distance between the pixel points having an amplitude value less than $50 \%$ of the average signal amplitude in this B-scan. However, irregularshaped inclusions cannot be sized by this procedure. The peak amplitude of the signal at a few discrete points in the air-coupled $\mathrm{C}$-scan had a very high amplitude, which is attributed to thickness resonance effects, and hence the $-6 \mathrm{~dB}$ drop method to size the defect in the C-scan image could not be adopted here. However, the dimensions of the inclusion from the C-scan image, shown in Figures 14(a) and 14(b), are estimated by measuring the width of the pixel points where the signal level drops to half of the average amplitude in that particular C-scan. This procedure for sizing the defects from the $\mathrm{C}$-scan gave reasonable agreement with actual sizes. Table 2 shows the area of inclusion estimated from the Lamb wave B-scan measurements with those obtained from the point-topoint through-transmission C-scan measurements. A comparison of defect locations obtained from the measurements by both techniques is summarised in Table 3.

Table 2. Comparison of the estimated size of the inclusions in the pipe using the air-coupled system

\begin{tabular}{|c|c|c|c|}
\hline Inclusion & $\begin{array}{c}\text { True size } \\
\left(\mathrm{mm}^{2}\right)\end{array}$ & $\begin{array}{c}\text { Inclusion size from } \\
\text { air-coupled through- } \\
\text { transmission C-scan } \\
\left(\mathrm{mm}^{2}\right)\end{array}$ & $\begin{array}{c}\text { Inclusion width from } \\
\text { Lamb wave B-scan } \\
(\mathrm{mm})\end{array}$ \\
\hline A & $15 \times 15$ & $17 \times 17$ & 13 \\
\hline B & $20 \times 20$ & $23 \times 23$ & 14 \\
\hline C & $25 \times 25$ & $25 \times 25$ & 19 \\
\hline D & $30 \times 30$ & $30 \times 30$ & 25 \\
\hline
\end{tabular}

Table 3. Comparisons of the estimated location of inclusions in the pipe using the air-coupled system

\begin{tabular}{|c|c|c|c|}
\hline Inclusion & $\begin{array}{c}\text { True distance } \\
\text { between } \\
\text { inclusions } \\
(\mathrm{mm})\end{array}$ & $\begin{array}{c}\text { Distance between } \\
\text { inclusions by air-coupled } \\
\text { through-transmission } \\
\text { C-scan }(\mathrm{mm})\end{array}$ & $\begin{array}{c}\text { Distance between } \\
\text { inclusions by } \\
\text { Lamb wave } \\
\text { B-scan }(\mathrm{mm})\end{array}$ \\
\hline A and B & 125 & 130 & 149 \\
\hline C and D & 90 & 85 & 102 \\
\hline
\end{tabular}

\subsection{Experimental results: conventional immersion- based C-scan}

The pipe was immersed in a water tank. A conventional immersion pulse-echo ultrasonic C-scan was carried out on the pipe, using a $2.25 \mathrm{MHz}$ immersion transducer, to validate the results from the air-coupled C-scan inspection system. An automated pipe scanner was used to generate a $\mathrm{C}$-scan image by axial translation of the transducer followed by rotation of the pipe centred on its axis. Inclusions present in the pipe wall prevent transmission and reflection of the ultrasonic pulse because of the acoustic impedance mismatch between the inclusions and the pipe. The peak signal amplitude of the reflected ultrasonic pulse was used to form the C-scan image shown in Figure 15. It is evident that the immersion $\mathrm{C}$-scan results shown in Figure 15 match reasonably well with the air-coupled C-scan shown in Figure 14.

\section{Summary and conclusion}

This paper has discussed the development of a Lamb wave-based inspection technique for the non-contact detection of inclusions in a filament-wound composite pipe. An air-coupled ultrasonic inspection system was set up to generate and receive the $\mathrm{A}_{0}$ Lamb wave mode in the pipe, along with automated data acquisition and imaging. A procedure for geometrically partitioning the pipe into various regions depending on the signal amplitude of the $\mathrm{A}_{0}$ mode was proposed for Lamb wave-based inspection. Experiments were carried out to generate Lamb wave-based B-scans to determine the approximate defect position in the pipe. Subsequently, an air-coupled through-transmission C-scan was carried out, only in the defective regions, to obtain more detailed information about the defect, thus reducing the time of inspection. All four artificial inclusions were detected using B-scans. The predicted sizes of the inclusion areas from the B-scans were found to be near to the actual sizes. The results reported here indicate that quick defect detection, position measurement and defect area sizing are feasible using a two-step exclusively non-contact inspection. Thus, this technique 


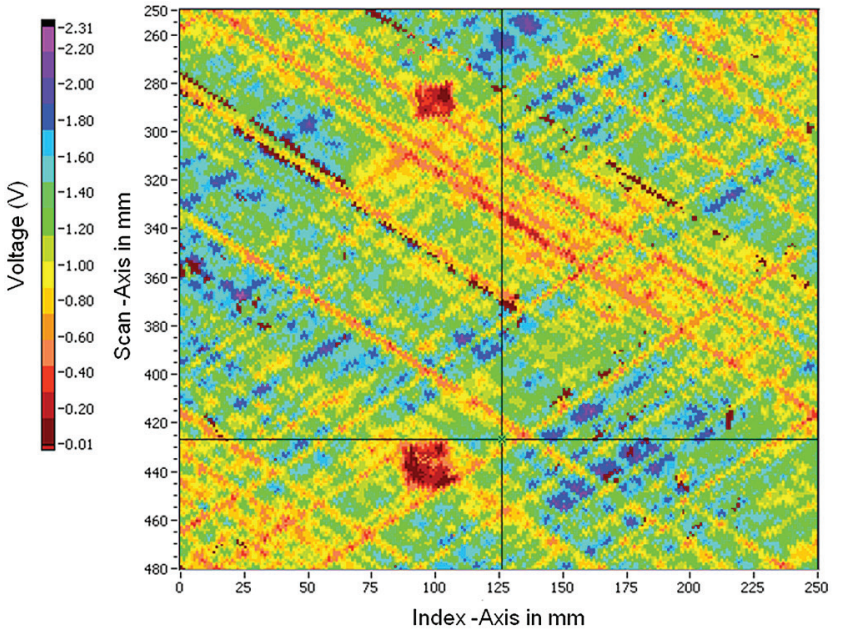

(a)

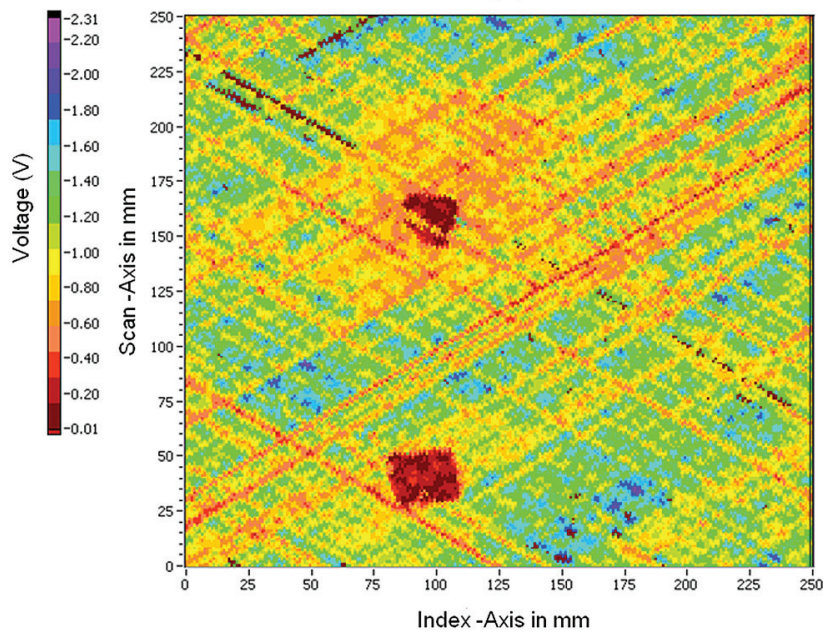

(b)

Figure 15. Pulse-echo $\mathrm{C}$-scans in the second segment of the pipe using a $2.25 \mathrm{MHz}$ immersion ultrasonic transducer: (a) region 1; and (b) region 2

firmly establishes that the proposed air-coupled non-contact inspection system has the potential to perform rapid pre-screening and replace traditional immersion-based inspection. However, further experiments are needed so that it can be extended to detect, locate and size other kinds of damage in the composite pipes. The limitation of this technique is that any misalignment in the optimum angle of the air-coupled transducer during Lamb wave scanning will adversely affect the received signal amplitude. Furthermore, the low-frequency air-coupled transducer, being inherently large in diameter, has an averaging effect on the signals received at the edges of defects. In conclusion, this work opens a new perspective for non-contact quick inspections of curved composite surfaces, such as long missile canisters and pressure tanks for civil and defence applications. Further improvement of this technique is being pursued with different sizes and frequencies of transducers, to make current through-transmission C-scan inspection an entirely single-sided approach.

\section{Acknowledgements}

The authors acknowledge the help rendered by Mr Ashok Kumar Mahankali for making the transducer attachments. The authors also thank Dr Mahesh Bhardwaj from Ultran Group for providing the GMP-based air-coupled transducers to IITM for this work. The research was funded by a grant from the NPMASS programme managed by ADA, Bangalore.

\section{References}

1. A R Martin, G F Fernando and K F Hale, 'Impact damage detection in filament-wound tubes using embedded optical fibre sensors', Smart Mater Struct, 6 (4), pp 470-476, 1997.

2. H K Kang, J S Park, D H Kang, C U Kim, C S Hong and C G Kim, 'Strain monitoring of a filament-wound composite tank using fibre Bragg grating sensors', Smart Mater Struct, 11 (6), pp 848-853, 2002.

3. M J S Lowe, D N Alleyne and P Cawley, 'Defect detection in pipes using guided waves', Ultrasonics, 36, pp 147-154, 1998.

4. J L Rose, D Jiao and J Spanner Jr, 'Ultrasonic guided wave NDE for piping', Mater Eval, 54 (11), pp 1310-1313, 1996.

5. W A Grandia and C M Fortunko, 'NDE applications of aircoupled ultrasonic transducers', IEEE Ultrasonics Symp Proc, 1, pp 697-709, 1995.

6. D W Schindel and D A Hutchins, 'Through-thickness characterisation of solids by wideband air-coupled ultrasound', Ultrasonics, 33 (1), pp 11-17, 1995.

7. R Stoessel, N Krohn, K Pfeiderer and G Busse, 'Air-coupled ultrasound inspection of various materials', Ultrasonics, 40, pp 159-163, 2002

8. M C Bhardwaj, 'Non-contact ultrasound - the final frontier in non-destructive analysis', Encyclopedia of Smart Materials, Ed: A Biderman, John Wiley \& Sons, New York, 2001.

9. D K Hsu, 'Non-destructive testing using air-borne ultrasound', Ultrasonics, 44 (1), pp e1019-e1024, 2006.

10. M Castaings and P Cawley, 'The generation, propagation and detection of Lamb waves in plates using air-coupled ultrasonic transducers', J Acoust Soc Am, 100, pp 3070-3077, 1996.

11. M Castaings, P Cawley, R Farlow and G Hayward, 'Singlesided inspection of composite materials using air-coupled ultrasound', J Non-destr Eval, 17, pp 37-45, 1998.

12. R Kazys, A Demcenko, E Zukauskas and L Mazeika, 'Aircoupled ultrasonic investigation of multi-layered composite materials', Ultrasonics, 44, pp 819-822, 2006.

13. R E Raišutis and E Zukauskas, 'Ultrasonic NDT of wind turbine blades using guided waves', Ultragarsas (Ultrasound), 63, pp 7-11, 2008.

14. M Castaings and B Hosten, 'Ultrasonic guided waves for health monitoring of high-pressure composite tanks', NDT\&E Intern, 41 (8), pp 648-55, 2008.

15. N Toyama and J Takatsubo, 'Lamb wave method for quick inspection of impact-induced delamination in composite laminates', Comp Sci Tech, 64, pp 1293-1300, 2004.

16. C Ramadas, J Padiyar, K Balasubramaniam, M Joshi and C V Krishnamurthy, 'Lamb wave-based ultrasonic imaging of interface delamination in a composite T-joint', NDT\&E International, 44, pp 523-530, 2010. 
2014-04-01

Lamb wave-based air-coupled ultrasonic inspection technique for filament-wound composite pipes

\section{Padiyar M, Janardhan}

British Institute of Non-destructive Testing

Padiyar M, Balasubramaniam K. (2014) Lamb wave-based air-coupled ultrasonic inspection technique for filament-wound composite pipes. Insight: Non-Destructive Testing \& Condition Monitoring, Volume 56, Issue 4, April 2014, pp. 195-202

https://doi.org/10.1784/insi.2014.56.4.195

Downloaded from Cranfield Library Services E-Repository 